\section{Implantação de rotinas de rastreamento do uso de risco de álcool e de uma intervenção breve na atenção primária à saúde: dificuldades a serem superadas}

\author{
Hazardous alcohol use: screening and brief \\ intervention as routine practice in primary care
}

Telmo Mota Ronzani 1,2

Mário Sérgio Ribeiro ${ }^{2}$

Michaela Bitarello do Amaral 1

Maria Lúcia Oliveira de Souza Formigoni 3

\footnotetext{
1 Instituto de Ciências

Humanas e de Letras, Universidade Federal de Juiz de Fora, Juiz de Fora, Brasil.

2 Faculdade de Medicina, Universidade Federal de Juiz de Fora, Juiz de Fora, Brasil.

3 Departamento de

Psicobiologia, Escola Paulista

de Medicina, Universidade

Federal de São Paulo,

São Paulo, Brasil.

Correspondência

M. L. O. S. Formigoni

Departamento de

Psicobiologia, Escola

Paulista de Medicina

Universidade Federal

de São Paulo.

Rua Botucatu 862,

São Paulo, SP

04023-062, Brasil.

mlformig@psicobio.epm.br
}

\begin{abstract}
The variety of problems related to the so-called hazardous patterns of alcohol use make the latter a prominent public health concern. Screening instruments associated with brief interventions in primary care provide one of the frequently recommended secondary prevention strategies. The objective of this study was to assess the implementation of this strategy as a routine practice in primary health care services in the city of Juiz de Fora, Minas Gerais State, Brazil. A qualitative design was employed, including the application of semi-structured interviews for managers and health care professionals in the city's public health system, in addition to content analysis and participant observation. Results point to difficulties in the effective implementation of such routines both by managers and health care professionals. Health care professionals limit the approach to alcohol-dependent patients and demonstrate lack of motivation for preventive work. Health services managers experience difficulties in the organization and administration of such instruments, despite affirming their interest in the project.
\end{abstract}

Alcohol Drinking, Primary Health Care, Prevention

\section{Introdução}

Além do problema da indução de dependência, o uso de álcool está relacionado a diversos problemas de saúde. Entre 20,0 e 58,0\% dos pacientes internados em hospitais gerais apresentam problemas relacionados ao uso de bebidas alcoólicas 1,2 . Ademais, estima-se que cerca de 5,0\% das mortes de pessoas entre 15 e 29 anos, em todo o mundo, estejam relacionadas ao uso de álcool 3. Estudos demonstram também uma alta prevalência de uso nocivo de álcool em pacientes que freqüentam serviços de atenção primária à saúde, atingindo cerca de $50,0 \%$ entre os homens e $40,0 \%$ entre as mulheres 4 . Apesar de não existirem dados conclusivos acerca do uso de risco ou nocivo de bebidas alcoólicas na população brasileira, um abrangente estudo nacional registrou taxa de $11,2 \%$ de dependentes de álcool na população geral do Brasil 5. Por tais razões, tornou-se evidente a necessidade de ações de prevenção secundária para o uso de risco de bebidas alcoólicas na atenção primária à saúde. Em diversas partes do mundo existe um interesse crescente de pesquisadores e gestores de saúde na implantação de tais ações 6 . Uma estratégia cada vez mais utilizada em diversos países tem sido a utilização de instrumentos de triagem associados a intervenções breves.

Uma importante ferramenta para a definição de estratégias de prevenção secundária são 
os instrumentos de rastreamento (screening). O principal instrumento de rastreamento para o uso de álcool atualmente em uso é o AUDIT (Alcohol Use Disorders Identification Test), um instrumento de auto-relato desenvolvido para identificar vários padrões de uso de álcool, de fácil aplicação e correção e com validação transcultural. Este instrumento é utilizado para ações de prevenção, realizadas em serviços de diferentes níveis e diferentes contextos 7. Quando associado à intervenção breve, o AUDIT facilita a aproximação inicial e permite um retorno (feedback) objetivo para o paciente, possibilitando assim a introdução dos procedimentos de intervenção breve e a motivação para a mudança de comportamento 8 .

Os estudos sobre a intervenção breve se concentram em duas direções principais: sua efetividade na redução de padrões de uso da substância e as condições em que tem sido implementada, focalizando especialmente o preparo dos profissionais envolvidos 9,10,11,12. Alguns estudos indicam que a utilização de apenas 5 a 10 minutos da consulta de rotina para aconselhamento dos usuários de risco de álcool, por profissionais de saúde consegue reduzir o consumo em 20,0-30,0\% 13. Diversos estudos experimentais têm produzido evidências acerca da eficácia de intervenções breves sobre o uso de risco de álcool em serviços de atenção primária à saúde $3,7,8$. Trabalhos de revisão sistemática concluíram que a intervenção breve apresenta resultados satisfatórios comparados aos de intervenções mais longas 14, ou ainda que as pessoas que recebiam as intervenções breves apresentavam o dobro de chances de diminuição do uso de álcool, comparadas a pessoas que não recebiam nenhuma intervenção 15. A efetividade de sua implementação, todavia, ainda carece de comprovação 6,9,11,12.

De acordo com Aalto \& Seppa 9 , dependendo da região, durante o período de um ano, entre 60,0 e $75,0 \%$ da população procuram algum tipo de atendimento em serviços de atenção primária à saúde. Por tal razão, a implantação de estratégias de intervenção breve para usuários de risco, nestes serviços, permitiria detectar pessoas com uso de risco, mas ainda não dependentes de álcool, que usualmente não procuram serviços especializados, e intervir precocemente.

De uma forma geral, a intervenção breve apresenta um enfoque educativo e motivacional dos pacientes para a redução do uso de álcool. É caracterizada pela sua curta duração, podendo variar de 5 a 60 minutos utilizando, em geral, entre uma e três sessões 8 .

Observa-se, entretanto, que existem dificuldades para a implementação deste tipo de intervenção na rotina dos serviços de atenção primária à saúde, como a falta de engajamento dos profissionais de saúde e a descrença pela possibilidade de melhora dos pacientes em relação a problemas associados ao uso de álcool. Além disso, o paradigma preventivo, embora amplamente discutido na teoria, raramente é colocado em prática nos serviços de saúde 16,17,18. Outra barreira constante na implantação de rotinas de prevenção ao uso abusivo de álcool seria a dificuldade dos gestores em ponderar adequadamente a importância de tais ações 19 .

Estratégias que visem mudança de atitudes, conhecimento e habilidades dos profissionais podem ser importantes na implementação de ações preventivas do uso abusivo de álcool. Alguns estudos demonstram que, quando os profissionais possuem atitudes positivas em relação ao usuário de álcool, sentem-se tecnicamente mais preparados para utilizar procedimentos de intervenção breve 9,11,15. Por esta razão, os programas de capacitação e treinamento de profissionais de atenção primária à saúde devem ser pragmáticos e enfocar a mudança de atitudes em relação aos usuários de álcool 20 .

Algumas ações de saúde pública, visando à redução do uso de risco de álcool, baseiam-se em estratégias populacionais. Estas não se restringem apenas ao enfoque individual, com objetivos pontuais e curativos, mas têm maior alcance e levam em consideração aspectos culturais das populações-alvo. Consistem sobretudo na disseminação de informações epidemiológicas e no desenvolvimento de estratégias de ação mais precisas, como o treinamento de profissionais na utilização de instrumentos de rastreamento e técnicas de intervenção breve 10 . Todavia, isoladamente, o treinamento não é capaz de mudar a prática dos profissionais nos serviços 11. É preciso alterar a organização do sistema de saúde e o entendimento, por parte dos gestores, da importância de se priorizar tais estratégias. Diferenças na estrutura e organização dos serviços e das práticas em saúde estão associadas a diferentes graus de comprometimento dos profissionais e gestores, em relação às práticas de prevenção e promoção 21,22.

Um modelo sistematizado de estratégias de disseminação de rotinas de rastreamento e prevenção do uso abusivo do álcool, já utilizado 
com sucesso em alguns países, foi proposto por Babor \& Higgins-Biddle 22 abrangendo três diferentes focos de ação: os profissionais da atenção primária à saúde, os gestores (locais, estaduais e federais) e a comunidade.

Tais estratégias de disseminação deveriam ser implementadas levando-se em consideração tanto o contexto cultural quanto os princípios da organização dos sistemas de saúde locais, buscando, entretanto, ir além das práticas tradicionais. Informações e estratégias devem ser claras, objetivas e pragmaticamente correlacionadas aos conceitos e práticas de saúde coletiva. Uma abordagem centrada nos efeitos biológicos individuais do uso de álcool, desligada do contexto e das crenças que os profissionais possam ter sobre tal uso, torna-se inadequada e/ou pouco efetiva do ponto de vista de sua aplicação à saúde coletiva. Para se atingirem mudanças abrangentes, torna-se também necessário o estabelecimento de alianças entre diversos setores da sociedade.

Os objetivos do presente trabalho foram avaliar as dificuldades encontradas durante um processo de treinamento de profissionais de saúde no rastreamento do uso de álcool, seguido por uma intervenção breve, e a sua implantação como uma nova rotina dos serviços de atenção primária à saúde em um município brasileiro.

\section{Metodologia}

\section{Local}

Juiz de Fora é um município brasileiro situado no Estado de Minas Gerais, com aproximadamente 480 mil habitantes. Sua rede assistencial é composta por 54 unidades básicas de saúde e 82 equipes de saúde da família, além de outros profissionais não vinculados ao Programa Saúde da Família (PSF), mas que também atendem aos pacientes no nível primário. No município, há uma sistematização do acesso aos serviços de saúde mental, com um protocolo de acolhimento, avaliação, referência e contra-referência dos pacientes que procuram os serviços de atenção primária. Todo paciente, para entrar na rede assistencial de saúde mental do município, deve ser avaliado inicialmente, em um dos serviços de atenção primária à saúde (unidades básicas de saúde/equipes de saúde da família), exceto pacientes atendidos pelo serviço de urgências psiquiátricas. Estudo realizado neste município encontrou taxas de $69,6 \%$ de uso-navida de bebidas alcoólicas e 11,0\% de sujeitos com provável diagnóstico de dependência 23.

\section{Participantes}

Participaram do projeto dois tipos de profissionais: cinco gestores do sistema municipal de saúde e quarenta profissionais que atuavam no atendimento de pacientes em serviços de atenção primária à saúde. Quatro gestores atuavam na Diretoria de Saúde, Saneamento e Desenvolvimento Ambiental da Prefeitura Municipal de Juiz de Fora e do Serviço de Atenção à Saúde do Servidor da Universidade Federal de Juiz de Fora e um no serviço de saúde do Corpo de Bombeiros. Estes profissionais foram selecionados com base na importância dos seus cargos de gestão na organização e implementação do projeto (amostra intencional). Os quarenta profissionais de saúde que atuavam em serviços de atenção primária foram os primeiros voluntários que responderam ao convite para participar de um treinamento sobre detecção do uso de álcool, seguida por intervenção breve (amostra de conveniência). O convite havia sido encaminhado a todos os profissionais de cada unidade de saúde pela Diretoria de Saúde, Saneamento e Desenvolvimento Ambiental de Juiz de Fora.

\section{Método}

\section{- Estratégia de divulgação do projeto}

Foi realizado inicialmente um trabalho de sensibilização da comunidade local para a importância da detecção precoce e práticas preventivas ao uso disfuncional de álcool. Neste sentido, a primeira ação foi dirigida aos gestores, profissionais de saúde e pessoas interessadas da comunidade, na forma de um seminário no qual foram resumidamente discutidos os problemas decorrentes do uso abusivo de álcool, sua prevalência e métodos para detecção precoce e intervenção breve. Em seguida foi apresentado o desenho do projeto e proposta sua implantação naquela cidade. Foram convidados para esse seminário todos os profissionais de atenção primária à saúde do município, técnicos do Programa de Atenção ao Dependente Químico, gerentes das unidades básicas de saúde, representantes do Conselho Municipal de Saúde, do Poder Judiciário, do Poder Legislativo, da Polícia Militar, das Associações de Bairro, da Associação Comercial e Industrial, da imprensa local (jornal, TV, rádio), além de professores, técnicos e alunos dos cursos da área de saúde da Universidade Federal de Juiz de Fora. Durante o seminário acima referido, os profissionais interessados em participar do treinamento inscreveram-se em uma lista de can- 
didatos, sendo selecionados preferencialmente aqueles pertencentes à mesma equipe. Antes de proceder ao treinamento do primeiro grupo, os pesquisadores visitaram os serviços de saúde para conhecer a realidade e a dinâmica de trabalho dos profissionais, com o objetivo de avaliar as expectativas sobre o projeto e o trabalho de prevenção.

\section{- Treinamento dos profissionais}

Os quarenta profissionais dos serviços de atenção primária à saúde participaram de um treinamento com carga horária de 16 horas, que teve como conteúdo: epidemiologia do uso de álcool no Brasil e aspectos biológicos, psicológicos e sociais; critérios para classificação do uso (risco, abuso ou dependência); instrumentos de diagnóstico e triagem; importância da prevenção ao uso abusivo de álcool na atenção primária; treinamento na aplicação do AUDIT (através da técnica de role-playing) e dos procedimentos de intervenção breve na atenção (role-playing); instruções sobre como implantar o AUDIT e a intervenção breve na rotina de atendimento. Foram treinados 40 profissionais, sendo 9 médicos, 14 enfermeiros, 8 assistentes sociais, 1 psicólogo e 8 auxiliares de enfermagem. A idade média era de 40 anos, sendo $80,0 \%$ do gênero feminino. O principal responsável, tanto pelo treinamento quanto pela avaliação, foi o primeiro autor deste trabalho, com a participação de dois outros pesquisadores e auxiliares de pesquisa devidamente treinados.

\section{- Avaliação do treinamento}

Para a avaliação da satisfação dos profissionais em relação ao treinamento foi aplicado um questionário estruturado com questões sobre avaliação do treinamento quanto à didática, conteúdo, aplicabilidade da proposta na prática, adequação da carga horária, havendo espaço para críticas e sugestões. Este instrumento foi respondido imediatamente após o término do treinamento, sendo despendidos $15 \mathrm{minu}$ tos em média para o seu preenchimento.

\section{- Entrevistas com os gestores}

Para se avaliar o grau de comprometimento e expectativas dos gestores em relação ao projeto, foi realizada uma entrevista semi-estruturada, com posterior análise de conteúdo - do tipo estrutural e temática, com delimitação prévia dos temas e subtemas abordados $24,25,26$. Os grandes temas abordados foram: (1) suas razões para participação; (2) suas expectativas em re- lação ao projeto; (3) suas expectativas quanto às contribuições dos pesquisadores; (4) sua possível contribuição ao projeto; (5) seus critérios para avaliação dos objetivos do projeto; (6) os prováveis obstáculos esperados; (7) possíveis estratégias a serem usadas para superar os obstáculos; (8) adequação do modelo proposto à rotina dos serviços; e (9) dificuldades para implantação da proposta na rotina de trabalho. As entrevistas foram realizadas em local isolado, após agendamento prévio com os gestores, tendo sido gravadas em fitas cassete para posterior transcrição e análise. Sua duração média foi de aproximadamente quarenta minutos. Todas as entrevistas foram realizadas pelo mesmo pesquisador e analisadas por dois pesquisadores com experiência na análise de dados qualitativos, sendo atingido consenso em todos os casos.

\section{- Processo de implantação do projeto}

A estratégia principal para viabilizar a implantação do projeto foi discutir com os gestores locais a forma mais adequada ao seu contexto organizacional, sendo os pesquisadores responsáveis pela supervisão e consultoria e os técnicos responsáveis pela forma de implantação. Após o treinamento, a forma de concretizar a implementação das rotinas de rastreamento e intervenção breve foi decidida com cada equipe das unidades de atenção primária à saúde, respeitando-se as peculiaridades e tipo de organização de cada serviço.

Durante todo o processo de implantação foi utilizada também a metodologia de observação participante dos pesquisadores durante as reuniões com os gestores e durante o treinamento e visitas aos serviços, sendo os dados anotados em caderno de campo, para posterior análise.

O projeto foi submetido e aprovado pelo Comitê de Ética em Pesquisa da Universidade Federal de São Paulo (0145/03). Todos os participantes do estudo assinaram o termo de consentimento livre e esclarecido em conformidade com as normas da instituição.

\section{Resultados}

Avaliação do impacto do seminário, realizada durante as visitas às unidades básicas

Sobre o encontro, os profissionais relataram que avaliaram negativamente a participação dos gestores, considerando que eles fizeram uso do evento para autopromoção política. Esta críti- 
ca corroborou outras observações que indicaram a existência de um descontentamento dos profissionais de saúde com a gestão da Diretoria de Saúde, Saneamento e Desenvolvimento Ambiental. Esta situação de insatisfação geral se dava tanto em relação a questões salariais e estruturação da carreira, quanto às condições de infra-estrutura dos locais de trabalho. A infra-estrutura dos serviços era heterogênea, existindo algumas unidades básicas de saúde com condições inadequadas e outras mais novas e modernas.

Apesar das críticas ao sistema, os profissionais manifestaram expectativas positivas sobre o treinamento, reconhecendo ser a questão do uso de álcool um problema com alta prevalência entre os pacientes por eles atendidos. Segundo os profissionais, foi a primeira vez que receberam treinamento focalizando a questão do uso abusivo de álcool, sendo o treinamento considerado oportuno. Além disso, consideraram que o trabalho por eles realizado até aquele momento, junto aos pacientes dependentes de álcool, necessitava de ser sistematizado e que o projeto proposto viabilizaria a sistematização do atendimento aos usuários disfuncionais de álcool, com ferramentas de trabalho úteis.

\section{Avaliação do impacto do treinamento, realizada durante o treinamento e nas visitas às unidades básicas}

Algumas observações foram feitas durante o treinamento para se analisar como foi o processo de mudança de atitude dos profissionais de saúde. No início, observamos uma resistência que foi sendo quebrada ao longo do treinamento. As principais resistências apresentadas resumem-se no desconforto ao perguntar sobre o hábito de consumo de álcool dos pacientes, particularmente nas áreas mais violentas do município. No entanto, à medida que os profissionais foram se familiarizando com a aplicação do AUDIT, perceberam que os pacientes recebem bem as perguntas e não se incomodam com este tipo de abordagem. O tema "dependência" se destacou durante as discussões realizadas no treinamento, indicando que os profissionais valorizavam pouco a questão da prevenção do uso de risco, apesar de o treinamento ter dado destaque à importância da prevenção e enfocado o uso de risco.

Durante as visitas às unidades básicas de saúde, e segundo o relato dos profissionais, percebeu-se que a maioria dos profissionais já realizavam algum tipo de intervenção breve; porém, sem sistematização ou critérios de inclu- são claramente definidos, sendo em geral dirigida apenas às pessoas com problemas evidentes quanto ao uso de álcool.

As respostas ao questionário aplicado ao final do treinamento estão sistematizadas na Tabela 1 . Todos os profissionais consideraram o treinamento bom ou excelente. Em relação ao conteúdo, a maioria das pessoas $(77,4 \%)$ também avaliou positivamente os temas abordados. As principais críticas, mencionadas por $16,2 \%$, dirigiram-se ao excesso de carga horária de epidemiologia (excesso de dados estatísticos) sobre o uso de álcool no Brasil e no mundo e à ênfase excessiva na classificação das zonas de risco do AUDIT.

Quanto aos aspectos didáticos, também houve uma aprovação expressiva (93,6\% de bom ou ótimo) dos participantes. A maioria dos profissionais $(93,5 \%)$ considerou o conteúdo programático aplicável na sua rotina de trabalho. Somente dois profissionais $(6,5 \%)$ avaliaram que o conteúdo teria uma aplicabilidade parcial. A carga horária do treinamento também foi considerada adequada. A principal sugestão ou crítica apresentada foi quanto à necessidade de um caráter mais prático do treinamento.

\section{Avaliação realizada um mês após o treinamento}

Um mês após o treinamento, foi feita uma nova visita nos serviços para se avaliar como os profissionais ou equipes estavam organizando a implantação da proposta. Houve relatos de que, visando à implantação imediata, o período do treinamento (novembro de 2003) foi inadequado, pois antecedeu à época de fechamento dos relatórios de produção anuais, um período de feriados prolongados e de férias de boa parte dos profissionais. Dessa forma, um mês após, várias equipes ainda não haviam iniciado, de fato, a aplicação da proposta na rotina.

As principais dificuldades apontadas pelos profissionais da atenção primária à saúde para a implantação do AUDIT e da intervenção breve na rotina foram: sobrecarga de trabalho das equipes, sendo estas majoritariamente incompletas; alta rotatividade dos profissionais; falta de motivação de algumas equipes decorrente da situação atual do sistema de saúde; menores níveis de motivação dos profissionais da equipe que não foram treinados; e dificuldades dos pacientes com baixo nível de escolaridade para responder ao AUDIT.

Além da dificuldade de se estabelecer uma parceria efetiva com os gestores locais, ao longo do mês de treinamento, os profissionais da 
atenção primária à saúde fizeram algumas críticas e reivindicações, relativas ao funcionamento do sistema de saúde mental. Ressaltaram a inadequada supervisão do nível secundário, na área de saúde mental, levando a limitações na sistematização do atendimento aos pacientes alcoolistas em algumas regiões, bem como na resolutividade desse atendimento. Os profissionais manifestaram maior interesse no conhecimento do protocolo de atendimento em saúde mental, que, embora existente há mais de cinco anos, ainda era desconhecido por alguns profissionais. Com base no projeto, os profissionais começaram a dar maior importância à hierarquização do atendimento de pacientes.

Em vista das críticas, algumas sugestões de estratégias foram propostas. Alguns profissionais da atenção primária à saúde sugeriram que a aplicação do AUDIT fosse realizada por todos os membros da equipe. Para isto, os agentes comunitários do PSF deveriam ser treinados, para depois ser realizado o encaminhamento dos pacientes com uso de risco ou dependência para um profissional de nível superior (médicos e enfermeiras).

As formas de aplicação do AUDIT e da Intervenção Breve, sugeridas pelas equipes de diferentes unidades básicas de saúde, foram bastante semelhantes. De uma forma geral, os profissionais propuseram que o AUDIT fosse aplicado nas visitas domiciliares, nas salas de espera, nos grupos de atendimento (hipertensão, diabetes etc.). Alguns serviços propuseram deixar o AUDIT no balcão de atendimento, visando à auto-aplicação pelos pacientes, e outros sugeriram sua inclusão nas consultas de rotina. A intervenção breve seria conduzida pelos profissionais da equipe que participaram do treinamento. No caso do serviço de saúde do Corpo de Bombeiros, decidiu-se que a aplicação do AUDIT e intervenção breve será incluída na avaliação de saúde anual dos recrutas. Sendo assim, todo o efetivo deverá ser submetido à aplicação do AUDIT e, desta forma, seria possível avaliar de maneira mais sistematizada o padrão de consumo de álcool destas pessoas.

\section{Entrevista com os gestores}

Foram abordadas, inicialmente, as razões pelas quais as instituições envolvidas concordaram em participar do projeto, sendo a principal razão o peso institucional que respaldava os pesquisadores, vinculados a centros de pesquisas reconhecidos nacionalmente e internacionalmente. Outra razão ressaltada foi a relevância da capacitação de profissionais de atenção primária à saúde para a detecção precoce do uso
Tabela 1

Avaliação do treinamento realizada pela aplicação de um questionário.

\begin{tabular}{lcr}
\hline Critérios e categorias & Freqüência & $\%$ \\
\hline 1. Avaliação geral & 19 & 61,3 \\
Bom & 12 & 38,7 \\
Ótimo & & \\
2. Avaliação do conteúdo & 2 & 6,5 \\
Adequado, mas pontos importantes & 4 & 12,9 \\
não foram abordados & & \\
Adequado, mas alguns pontos foram & 20 & 64,5 \\
excessivamente detalhados & 4 & 12,9 \\
Muito bom & 1 & 3,2 \\
Ótimo & & \\
Não respondeu & & 83,9 \\
3. Temas muito abordados & 26 & 9,7 \\
Sem críticas & 3 & 6,5 \\
Epidemiologia & 2 &
\end{tabular}

4. Temas pouco abordados

Sem críticas

Dependência

Família

93,5

3,2

3,2

5. Avaliação da apresentação

Razoável

Boa

Excelente

6. Avaliação da aplicabilidade

Parcial

Boa

Excelente

7. Avaliação da carga horária

Carga horária muito pequena

Carga horária deveria ser um pouco maior

Carga horária adequada

Carga horária poderia ser um pouco menor

Carga horária excessiva

\section{Sugestões e críticas}

Sem críticas ou sugestões

Conteúdos mais práticos

Mais membros da mesma equipe

Motivação dos profissionais

Educação continuada

Intervalo quinzenal dos módulos

Treinamento do agente comunitário de saúde

Treinamento no início da semana

38,7
29,0
12,9
6,5
3,2
3,2
3,2
3,2
100,0


de álcool, tendo em vista a alta prevalência de uso de risco.

Quanto à expectativa em relação ao projeto, foi ressaltado que a implantação do projeto poderia aumentar a resolutividade dos serviços, em relação aos casos de uso abusivo de álcool. Foi considerada também a possível contribuição do projeto na sistematização dos serviços, com o fornecimento de ferramentas de trabalho úteis, tendo sido destacada a necessidade de supervisão do trabalho dos profissionais.

Quanto às contribuições e/ou retornos que os gestores esperavam da equipe de pesquisa, foi ressaltada a necessidade de avaliação do processo inicial de implantação, incluindo um retorno (feedback) objetivo sobre os resultados alcançados. Outras contribuições esperadas foram a melhoria na integração de ações preventivas com as desenvolvidas nos diversos níveis de atenção e a avaliação da satisfação dos profissionais treinados.

Em relação às contribuições que os parceiros poderiam dar ao projeto, observou-se uma reação inicial de indefinição sobre o tema. Quando estimulados a responder, as contribuições sugeridas foram a participação ativa no processo de implantação, o trabalho de motivação dos profissionais, a cobrança do envolvimento de gestores de níveis superiores e a garantia de continuidade da proposta a médio prazo.

O principal critério de avaliação dos resultados do projeto proposto pelos gestores foi a mensuração dos níveis de resolutividade das equipes treinadas, por exemplo, pela futura diminuição da demanda de pacientes com problemas por uso de álcool que se dirigem a serviços especializados. Outro critério de avaliação apontado foi a incorporação do modelo nos serviços, como indicador de sua adequação à realidade local e da satisfação dos profissionais em relação à proposta de trabalho.

Possíveis obstáculos à execução do projeto também foram abordados, entre eles a pouca motivação dos profissionais, que foi constatada nas visitas aos serviços, como já mencionado. As sugestões de estratégias para superação de tais obstáculos, apesar de pertinentes, foram pouco objetivas. Destacaram-se as mudanças no plano macropolítico, a redefinição de políticas de saúde no sentido de uma priorização de fato das ações de atenção primária à saúde e a procura de uma maior integração dos níveis de atenção. Uma estratégia mais localizada para o município voltou-se para perspectiva de aumentar a motivação dos profissionais.

Quanto à adequação do modelo proposto à realidade local, todos os entrevistados mani- festaram sua crença na adequação teórica do modelo, tendo em vista sua coerência em relação aos princípios gerais da atenção primária à saúde. Sobre possíveis dificuldades na implantação da proposta como rotina diária nos serviços, os entrevistados referiram a falta de motivação e deficiência na formação dos profissionais de atenção primária à saúde. Sugeriram como estratégias para contornar tais dificuldades a implantação de um sistema de supervisão contínua e o retorno (feedback) dos resultados aos profissionais. Mencionaram também, a necessidade de uma mudança ampla na organização do sistema de saúde.

\section{Discussão}

A rigor, os resultados deste estudo sugerem que a implantação, em serviços públicos de atenção primária, de rotinas de rastreamento do uso de risco de álcool e posterior intervenção breve necessita, ao menos, levar em conta dois aspectos fundamentais ao entendimento de sua dinâmica: (1) o contexto de valores, atitudes e normas sociais relativos às práticas de consumo de substâncias psicoativas na população envolvida; e (2) a complexa relação entre técnicos e gestores dos serviços de saúde.

Assim sendo, a dificuldade inicial dos profissionais em abordar o consumo de bebidas alcoólicas dos usuários destes serviços aponta para os conflitos entre idéias e valores que envolvem os comportamentos associados ao uso de substâncias, lícitas e ilícitas, mais adequadamente discutidos por meio da contribuição antropológica 27.

Apesar de os treinandos terem verbalizado sua receptividade ao modelo transmitido - detecção do uso abusivo de álcool acoplado a uma técnica de intervenção breve -, foi verificado que sua implantação, de fato, não ocorreu de imediato nos serviços de atenção primária à saúde. Este estudo identificou, dentre as principais dificuldades para a implantação da proposta, o despreparo dos profissionais para atuarem em ações preventivas, o subdimensionamento dos recursos humanos e materiais à demanda existente, a falta de estímulo e motivação para o trabalho, a falta de integração dos diversos níveis de atenção e problemas no planejamento e organização do sistema de saúde. A efetiva participação dos gestores também seria fundamental para uma implantação bem sucedida e para a se atingir a resolutividade esperada da proposta de trabalho, favorecendo sua incorporação à rotina assistencial do sistema de saúde local. 
Além das dificuldades relativas à própria formação dos profissionais e seus conceitos acerca do processo saúde-doença, as práticas de saúde coletiva são circunscritas por múltiplos determinantes ideológicos e interesses conflitantes, especialmente no que se refere ao poder político e interesses corporativos e empregatícios. Assim sendo, tais relatos e observações remetem a embates mais amplos e complexos, que ultrapassam a questão imediata da implantação de novas rotinas assistenciais 27 .

A análise do processo detectou várias dificuldades a serem transpostas. Objetivamente, contudo, diversas ações visando à minimização das dificuldades já foram iniciadas, em especial por meio de uma maior aproximação com os níveis intermediários dos gestores da Secretaria Municipal de Saúde. Entramos em contato com a coordenação da atenção primária e iniciamos uma parceria mais direta, visando sua maior participação. Além de expor à chefia do Departamento de Saúde Mental as sugestões e reivindicações dos profissionais da atenção primária à saúde, a equipe de pesquisadores procurou contribuir para uma maior interação entre as chefias de saúde mental e da atenção primária.

Vale ressaltar ainda que, além do problema da inadequação da formação do profissional de atenção primária à saúde no Brasil, bem como sua falta de identificação com o trabalho de prevenção e promoção em saúde, o clima organizacional não tem contribuído para motivar os profissionais de atenção primária à saúde. Foi observado, tanto durante o treinamento como ao longo das visitas aos serviços, que os técnicos de atenção primária à saúde se encontravam muito insatisfeitos com a política salarial, a precariedade das condições de trabalho, a organização do sistema de saúde e com a falta de resolutividade dos níveis secundários e terciários.

\section{Considerações finais}

O objetivo do presente trabalho foi descrever as dificuldades observadas no processo de implantação, visando a propor estratégias que permitam superar os entraves encontrados. Sabese que a fase inicial é fundamental para o sucesso a médio ou longo prazo de qualquer proposta. Por esta razão, a detecção precoce dos problemas e dificuldades é fundamental para o delineamento de estratégias que possam minimizar ou eliminar tais dificuldades.

Ressalta-se que o problema de se implantar ações preventivas não ocorre somente em relação ao uso abusivo de álcool, mas é comum a todos os problemas de saúde passíveis de prevenção. Apesar de, na teoria, a prioridade dos serviços de atenção primária à saúde ser a prevenção e a promoção em saúde, nota-se que, na prática, o trabalho individual e curativo ainda é preponderante nos serviços. Este paradigma gera enormes dificuldades na implantação de programas de prevenção, como o aqui mencionado. Torna-se, portanto, essencial um processo de acompanhamento e avaliação contínua, que permita a obtenção de resultados mais conclusivos.

\section{Resumo}

Tendo em vista os diversos problemas associados ao assim chamado uso de risco de álcool, ele é considerado uma relevante questão de saúde pública. Uma das estratégias de prevenção secundária recomendadas éa utilização de instrumentos de rastreamento associados a intervenções breves em serviços de atenção primária à saúde. O objetivo deste estudo foi avaliar o processo de implantação desta estratégia na rotina de atenção primária à saúde da cidade de Juiz de Fora, Minas Gerais, Brasil. Foi utilizada uma abordagem qualitativa, incluindo a aplicação de entrevistas semiestruturadas a gestores elou profissionais da assistência do Sistema Municipal de Saúde, associadas à análise de conteúdo e observação participante. Os resulta- dos indicam que há dificuldades para a implantação efetiva destas rotinas, tanto em relação aos gestores quanto aos profissionais envolvidos diretamente na sua execução. Quanto aos profissionais destacam-se a restrição da abordagem a dependentes de álcool e a falta de motivação dos profissionais para trabalhos preventivos. Quanto aos gestores, foram detectadas dificuldades práticas no processo de organização e gerenciamento, a despeito de um afirmado interesse no projeto.

Consumo de Bebidas Alcoólicas; Atenção Primária à Saúde; Prevenção 


\section{Colaboradores}

T. M. Ronzani e M. S. Ribeiro participaram do planejamento, trabalho de campo, análise dos dados e redação do artigo. M. L. O. S. Formigoni participou do planejamento, análise dos dados e redação do artigo. M. B. Amaral participou da coleta de dados, análise e redação do artigo.

\section{Agradecimentos}

Agradecemos o apoio financeiro da Associação Fundo de Incentivo à Psicofarmacologia (AFIP), ao Conselho Nacional de Desenvolvimento Científico e Tecnológico (CNPq), à Organização Mundial da Saúde e ao Projeto de Incentivo às Mudanças Curriculares dos Cursos de Graduação de Medicina (PROMED)/Universidade Federal de Juiz de Fora (UFJF). Agradecemos, ainda, a colaboração dos alunos da UFJF: Michaela Bitarello do Amaral, Priscila Montianélle de Castro, Molise de Bem Magnabosco, Glaucia Veigas, Juliana Torres Moraes e Danielle de Carvalho Machado que participaram da fase de coleta de dados.

Este trabalho faz parte de um estudo multicêntrico internacional (Evaluating Alcohol Brief Intervention Implementation), apoiado financeiramente pelo NIH/NIAAA-R21, coordenado por Thomas F. Babor e John C. Higgins-Biddle, do Department of Community Medicine Care \& Health Care, University of Connecticut Health Center, que participaram do planejamento inicial do projeto.

\section{Referências}

1. Moreira LFS, Masur J. Consumo de álcool em pacientes ambulatoriais de hospital geral na capital e interior do Estado do Rio Grande do Norte. Rev Assoc Bras Psiquiatr 1980; 2:183-9.

2. Masur J, Laranjeira R. Hipertensão e alcoolismo. Rev Assoc Med Bras 1980; 26:86-8.

3. Foxcroft DR, Ireland D, Lister-Sharp DJ, Lowe G, Breen R. Longer-term primary prevention for alcohol misuse in young people: a systematic review. Addiction 2003; 98:397-411.

4. Aalto M, Seppa K, Kiianmaa K, Sillanaukee P. Drinking habits and prevalence of heavy drinking among primary health care outpatients and general population. Addiction 1999; 94:1371-9.

5. Carlini EA, Galduróz JCF, Noto AR, Nappo SA. I Levantamento domiciliar sobre o uso de drogas no Brasil. São Paulo: Centro Brasileiro de Informações sobre Drogas Psicotrópicas; 2001.

6. Babor T, Caetano R, Casswell S, Edwards G, Giesbrecht N, Graham K, et al. Alcohol: no ordinary commodity - research and public policy. Oxford: Oxford University Press; 2003.

7. Babor T, Higgins-Biddle JC. Brief Intervention for harzardous and harmful drinking. Geneva: World Health Organization; 2001.

8. Babor T, Higgins-Biddle JC, Saunders JB, Monteiro MG. AUDIT - the alcohol use disorders identification test: guidelines for use in primary care. Geneva: World Health Organization; 2001.

9. Aalto M, Seppa K. Primary health care nurses' and physicians' attitudes, knowledge and beliefs regarding brief intervention for heavy drinkers. Addiction 2001; 96:305-11.

10. Caetano R, Cunradi C. Alcohol dependence: a public health perspective. Addiction 2002; 97:63345.

11. Bendtsen P, Akerlind I. Changes in attitudes and practices in primary health care with regard to early intervention for problem drinkers. Alcohol Alcohol 1999; 34:795-800.

12. Andreasson S, Hjalmarsson K, Rehnman C. Implementation and dissemination of methods for prevention of alcohol problems in primary health care: a feasibility study. Alcohol Alcohol 2000; 35:525-30.

13. Roche AM, Parle MD, Stubbs JM, Hall W, Saunders JB. Management and treatment efficacy of drug and alcohol problems: what do doctors believe? Addiction 1995; 90:1357-66.

14. Bien T, Miller WR, Toningan JS. Brief interventions for alcohol problems: a review. Addiction 1993; 88:315-36.

15. Wilk A, Jensen N, Havighusrt T. Meta-analysis of randomized control trials addressing brief interventions with problem drinkers. J Gen Intern Med 1997; 12:274-83.

16. Ronzani TM, Stralen CJ. Dificuldades de implantação do Programa de Saúde da Família como estratégia de reforma do sistema de saúde brasileiro. Revista de Atenção Primária à Saúde 2003; 6:99-107.

17. Ronzani TM, Ribeiro MS. Identidade e formação profissional dos médicos. Rev Bras Educ Méd 2003; $27: 229-36$ 
18. Richmond R, Mendelsohn C, Kehoe L. Family physicians' utilization of a brief smoking cessation program following reinforcement contact after training: a randomized trial. Prev Med 1998; 27:77-83.

19. Lock CA, Kaner E, Lamont S, Bond S. A qualitative study of nurses' attitudes and practices regarding brief alcohol intervention in primary health care. J Adv Nurs 2002; 39:333-42.

20. Lopez-de-Munain J, Torcal J, Lopez V, Garay J. Prevention in routine general practice: activity patterns and potential promoting factors. Prev Med 2001;32:13-22.

21. Lock CA, Kaner EF. Use of marketing to disseminate brief alcohol intervention to general practitioners: promoting health care interventions to health promoters. J Eval Clin Pract 2000; 6:345-57.

22. Babor T, Higgins-Biddle JC. Alcohol screening and brief intervention: dissemination strategies for medical practice and public health. Addiction 2000; 95:677-86.
23. Ribeiro MS, Vargas EV, Alves MJM, Guimarães LS, Moreira GM. O consumo de substâncias psicoativas em Juiz de Fora-MG. J Bras Psiquiatr 1999; 48:405-13.

24. Bardin L. Análise de conteúdo. Lisboa: Edições 70; 1977.

25. Minayo MCS. O desafio do conhecimento. Pesquisa qualitativa em saúde. São Paulo: Editora Hucitec/Rio de Janeiro: ABRASCO; 2000.

26. Turato ER. Tratado da metodologia da pesquisa clínico-qualitativa. Construção teórico-epistemológica, discussão comparada e aplicação nas áreas da saúde e humanas. Petrópolis: Editoras Vozes; 2003.

27. Neves DP. Alcoolismo: acusação ou diagnóstico? Cad Saúde Pública 2004; 20:7-14.

Recebido em 30/Jun/2004

Versão final reapresentada em 17/Jan/2005

Aprovado em 27/Jan/2005 